\title{
Backward Bifurcation of an Epidemic Model with Infectious Force in Infected and Immune Period and Treatment
}

\author{
Yakui Xue and Junfeng Wang \\ Department of Mathematics, North University of China, Shanxi Taiyuan 030051, China \\ Correspondence should be addressed to Yakui Xue, xyk5152@163.com
}

Received 18 January 2012; Accepted 27 May 2012

Academic Editor: Malisa R. Zizovic

Copyright (c) 2012 Y. Xue and J. Wang. This is an open access article distributed under the Creative Commons Attribution License, which permits unrestricted use, distribution, and reproduction in any medium, provided the original work is properly cited.

An epidemic model with infectious force in infected and immune period and treatment rate of infectious individuals is proposed to understand the effect of the capacity for treatment of infective on the disease spread. It is assumed that treatment rate is proportional to the number of infective below the capacity and is constant when the number of infective is greater than the capacity. It is proved that the existence and stability of equilibria for the model is not only related to the basic reproduction number but also the capacity for treatment of infective. It is found that a backward bifurcation occurs if the capacity is small. It is also found that there exist bistable endemic equilibria if the capacity is low.

\section{Introduction}

Recently, mathematical models describing the dynamics of human infectious diseases have played an important role in the disease control in epidemiology. Researchers have proposed many epidemic models to understand the mechanism of disease transmission. We assume that a susceptible individual first goes through a latent period after infection before becoming infectious. The resulting models are of SEI, SEIR, or SEIRS type, respectively. Zhang and Ma [1] studied the global stability of an SEI model with general contact rate. Yuan et al. [2] considered the local stability of the model having infectious force in both latent period and infected period. Li and Jin [3-5] studied the global stability of the epidemic model having infectious force in both latent period and infected period. Usually, these classical epidemic models have only one endemic equilibrium when the basic reproduction number $R_{0}>1$, and the disease-free equilibrium is always stable when $R_{0}<1$ and unstable when $R_{0}>1$. So the bifurcation leading from a disease-free equilibrium to an endemic equilibrium is forward. 
But in recent years, the phenomenon of the backward bifurcations has arisen the interests in disease control (see [6-15]). In this case, the basic reproduction number cannot describe the necessary disease elimination effort any more. Thus, it is important to identify backward bifurcations and establish thresholds for the control of diseases.

In classical epidemic models, the treatment rate of the infective is assumed to be proportional to the number of the infective. Because the resources of treatment should be limited, every community should have a suitable capacity for treatment. This hypothesis is satisfactory when the number of the infective is small and the resources of treatment are enough and unsatisfactory when the number of the infective is large and the resources of treatment are limited. Thus, it is important to determine a suitable capacity for the treatment of a disease. A constant treatment rate of disease is adopted in [16]. Note that a constant treatment rate is suitable when the number of infective is large. In [17], the treatment rate of the disease is modified into

$$
T(I)= \begin{cases}r I & \text { if } 0 \leqslant I \leqslant I_{0} \\ k & \text { if } I>I_{0}\end{cases}
$$

where $k=r I_{0}, r$ and $I_{0}$ are positive constant. This means that the treatment rate of disease is proportional to the number of the infective when the capacity of treatment is not reached and, otherwise, takes the maximal capacity. This improves the classical proportional treatment and the constant treatment in [16].

In this paper, we study the backward bifurcation and global dynamics of an epidemic model with infectious force in infected and immune period and treatment function. To formulate our model, we will consider a population that is divided into three types: susceptible, infective, and recovered. Let $S(t), I(t)$, and $R(t)$ denote the numbers of susceptible, infective, recovered individuals at time $t$, respectively. The total population size at time $t$ is denoted by $N(t)$.

The basic assumptions in the paper are as the follows.

(i) There is a positive constant recruitment rate of the population $A$.

(ii) Positive constant $d$ is the nature death rate of population.

(iii) $\beta_{1}, \beta_{2}$ are the rate of the efficient contact in the infected and recovered period, respectively.

(iv) Positive constant $\gamma$ is the natural recovery rate of infective individuals.

(v) Positive constant $\epsilon$ is the disease-related death rate.

(vi) The treatment of a disease is $T(I)$ in (1.1).

form:

Under the assumptions above, an epidemic model to be studied takes the following

$$
\begin{gathered}
\frac{d S}{d t}=A-d S-\beta_{1} S I-\beta_{2} S R, \\
\frac{d I}{d t}=\beta_{1} S I+\beta_{2} S R-(d+\gamma+\epsilon) I-T(I), \\
\frac{d R}{d t}=\gamma I+T(I)-d R,
\end{gathered}
$$


where $S(t)+I(t)+R(t)=N(t)$. It is easy to verify that $R_{+}^{3}$ is positive invariant for system (1.2).

According to $S(t)+I(t)+R(t)=N(t)$ and (1.1), $N(t)$ satisfies the following equation:

$$
\frac{d N}{d t}=A-d N-\epsilon I
$$

Then system (1.2) is equivalent to

$$
\begin{gathered}
\frac{d N}{d t}=A-d N-\epsilon I, \\
\frac{d I}{d t}=\left(\beta_{1} I+\beta_{2} R\right)(N-I-R)-(d+\gamma+\epsilon) I-T(I), \\
\frac{d R}{d t}=\gamma I+T(I)-d R .
\end{gathered}
$$

It is easy to verify that all solutions of system (1.4) initiating in set $\{(N, I, R) \mid N>$ $0, I \geqslant 0, R \geqslant 0, I+R \leqslant N\}$ eventually enter the set $\Omega=\{(N, I, R) \mid 0<N \leqslant A / d, I \geqslant 0, R \geqslant$ $0, I+R \leqslant N\}$. Therefore, $\Omega$ is positively invariant for system (1.4). We consider the solutions of system (1.4) in $\Omega$ below.

When $0 \leqslant I \leqslant I_{0}$, system (1.4) becomes

$$
\begin{gathered}
\frac{d N}{d t}=A-d N-\epsilon I, \\
\frac{d I}{d t}=\left(\beta_{1} I+\beta_{2} R\right)(N-I-R)-(d+\gamma+\epsilon+r) I, \\
\frac{d R}{d t}=(\gamma+r) I-d R .
\end{gathered}
$$

When $I>I_{0}$, system (1.4) becomes

$$
\begin{gathered}
\frac{d N}{d t}=A-d N-\epsilon I, \\
\frac{d I}{d t}=\left(\beta_{1} I+\beta_{2} R\right)(N-I-R)-(d+\gamma+\epsilon) I-k, \\
\frac{d R}{d t}=\gamma I+k-d R .
\end{gathered}
$$

The purpose of this paper is to show that system (1.4) has a backward bifurcation if the capacity for treatment is small. We obtain the sufficient conditions that the diseasefree equilibrium and endemic equilibria of system (1.4) are stable. It is shown that (1.4) has bistable endemic equilibria if the capacity is small. The organization of this paper is as follows. In next section, we study the existence and bifurcations of equilibria for (1.4). We analyze the stability of equilibria for (1.4) and present the numerical simulations in Section 3. 


\section{The Existence of Equilibria}

In this section, we consider the equilibria of system (1.4). Obviously, $E_{0}(A / d, 0,0)$ is the disease-free equilibrium of (1.4). For the endemic equilibrium $E(N, I, R)$ of $(1.4), N, I$ and $R$ satisfy

$$
\begin{gathered}
A-d N-\epsilon I=0, \\
\left(\beta_{1} I+\beta_{2} R\right)(N-I-R)-(d+\gamma+\epsilon) I-T(I)=0, \\
\gamma I+T(I)-d R=0 .
\end{gathered}
$$

When $0 \leqslant I \leqslant I_{0}$, system (2.1) becomes

$$
\begin{gathered}
A-d N-\epsilon I=0, \\
\left(\beta_{1} I+\beta_{2} R\right)(N-I-R)-(d+\gamma+\epsilon+r) I=0, \\
(\gamma+r) I-d R=0 .
\end{gathered}
$$

When $I>I_{0}$, system (2.1) becomes

$$
\begin{gathered}
A-d N-\epsilon I=0, \\
\left(\beta_{1} I+\beta_{2} R\right)(N-I-R)-(d+\gamma+\epsilon) I-k=0, \\
\gamma I+k-d R=0 .
\end{gathered}
$$

Form (2.2), I satisfies the following equation:

$$
\left(\beta_{1}+\beta_{2} \frac{\gamma+r}{d}\right) \frac{A-(d+\epsilon+\gamma+r) I}{d}=d+\epsilon+\gamma+r
$$

Therefore, we obtain

$$
I=\frac{A-d(d+\epsilon+\gamma+r) /\left(\beta_{1}+\beta_{2}((\gamma+r) / d)\right)}{d+\epsilon+\gamma+r} .
$$

Let

$$
R_{0}=\frac{A\left(\beta_{1}+\beta_{2}((\gamma+r) / d)\right)}{d(d+\epsilon+\gamma+r)} .
$$


Then $R_{0}$ is a basic reproduction number of (1.4). If $R_{0}>1$, then $I>0 ;(2.2)$ admits a unique positive solution $E_{*}=\left(N_{*}, I_{*}, R_{*}\right)$, where

$$
\begin{gathered}
N_{*}=\frac{A-\epsilon I_{*}}{d}, \\
I_{*}=\frac{A-d(d+\epsilon+\gamma+r) /\left(\beta_{1}+\beta_{2}((\gamma+r) / d)\right)}{d+\epsilon+\gamma+r}, \\
R_{*}=\frac{(\gamma+r) I_{*}}{d} .
\end{gathered}
$$

Clearly, $E_{*}$ is an endemic equilibrium of (1.4) if and only if

$$
1<R_{0} \leqslant 1+\frac{\beta_{1}+\beta_{2}((\gamma+r) / d)}{d} I_{0}
$$

According to (2.3), I satisfies the following equation:

$$
a_{0} I^{2}+a_{1} I+a_{2}=0
$$

where $a_{0}=\left(\beta_{1}+\beta_{2}(\gamma / d)\right)(d+\epsilon+\gamma)$,

$$
\begin{gathered}
a_{1}=d(d+\epsilon+\gamma)+\beta_{2} \frac{k}{d}(d+\epsilon+\gamma)-\left(\beta_{1}+\beta_{2} \frac{\gamma}{d}\right)(A-k), \\
a_{2}=d k-\beta_{2} \frac{k}{d}(A-k) .
\end{gathered}
$$

We only consider the case of $a_{2}>0$. If $a_{1} \geqslant 0$, it is clear that (2.9) does not have positive real root. Let us suppose $a_{1}<0$ below. Note that $a_{1}<0$ is equivalent to

$$
R_{0} \geqslant 1+\frac{\beta_{2}(k / d)(d+\epsilon+\gamma)+\beta_{2}(r / d) A+\left(\beta_{1}+\beta_{2}(\gamma / d)\right) k-d r}{d(d+\epsilon+\gamma+r)}=: p_{*}
$$

It is easy that

$$
\begin{aligned}
\Delta= & a_{1}^{2}-4 a_{0} a_{2}=R_{0}^{2} d^{2}(d+\epsilon+\gamma+r)^{2} \\
& -2 R_{0} d(d+\epsilon+\gamma+r)\left[\left(d+\beta_{2} \frac{k}{d}\right)(d+\epsilon+\gamma)+\beta_{2} \frac{r}{d} A+\left(\beta_{1}+\beta_{2} \frac{\gamma}{d}\right) k\right] \\
& +\left[\left(d+\beta_{2} \frac{k}{d}\right)(d+\epsilon+\gamma)+\beta_{2} \frac{r}{d} A+\left(\beta_{1}+\beta_{2} \frac{\gamma}{d}\right) k\right]^{2} \\
& -4\left(\beta_{1}+\beta_{2} \frac{\gamma}{d}\right)(d+\epsilon+\gamma)\left(d k-\beta_{2} \frac{k}{d}(A-k)\right) .
\end{aligned}
$$


It follows that $\Delta \geqslant 0$ is equivalent to

$$
R_{0} \geqslant p_{*}+\frac{2 \sqrt{\left(\beta_{1}+\beta_{2}(\gamma / d)\right)(d+\epsilon+\gamma)\left[d k-\beta_{2}(k / d)(A-k)\right]}}{d(d+\epsilon+\gamma+r)}=: p_{0}
$$

or

$$
R_{0} \leqslant p_{*}-\frac{2 \sqrt{\left(\beta_{1}+\beta_{2}(\gamma / d)\right)(d+\epsilon+\gamma)\left[d k-\beta_{2}(k / d)(A-k)\right]}}{d(d+\epsilon+\gamma+r)} .
$$

Thus $a_{1}<0$ and $\Delta \geqslant 0$ if and only if (2.13) holds. Let us suppose that (2.13) holds. Then (2.9) has two positive solutions $I_{1}$ and $I_{2}$ where

$$
I_{1}=\frac{-a_{1}-\sqrt{\Delta}}{2 a_{0}}, \quad I_{2}=\frac{-a_{1}+\sqrt{\Delta}}{2 a_{0}} .
$$

Set $N_{i}=\left(A-\epsilon I_{i}\right) / d, R_{i}=\left(\gamma I_{i}+k\right) / d$ and $E_{i}\left(N_{i}, I_{i}, R_{i}\right)(i=1,2)$. If $I_{i}>I_{0}(i=1,2)$, then $E_{i}$ is an endemic equilibrium of (1.6).

By the definition of $I_{1}$, we notice that $I_{1}>I_{0}$ is equivalent to

$$
-\sqrt{\Delta}>2 a_{0} I_{0}+a_{1}
$$

This implies that $2 a_{0} I_{0}+a_{1}<0$. By immediate calculation, $2 a_{0} I_{0}+a_{1}<0$ is equivalent to

$$
R_{0}>p_{*}+\frac{2\left(\beta_{1}+\beta_{2}(\gamma / d)\right)(d+\epsilon+\gamma) I_{0}}{d(d+\epsilon+\gamma+r)}=: p_{1}
$$

Further, $I_{1}>I_{0}$ demands that

$$
\left(2 a_{0} I_{0}+a_{1}\right)^{2}>\Delta
$$

By immediate calculation,

$$
R_{0}<1+\frac{\beta_{1}+\beta_{2}((\gamma+r) / d)}{d} I_{0}=: p_{2}
$$

Therefore, $I_{1}>I_{0}$ holds if and only if $R_{0}>p_{1}$ and $R_{0}<p_{2}$.

By similar discussions as previously mentioned, we have that $I_{2}>I_{0}$ holds if and only if either $R_{0}>p_{1}$, or $R_{0}>p_{2}, R_{0}<p_{1}$.

Summarizing the discussions above, we have the following conclusion. 


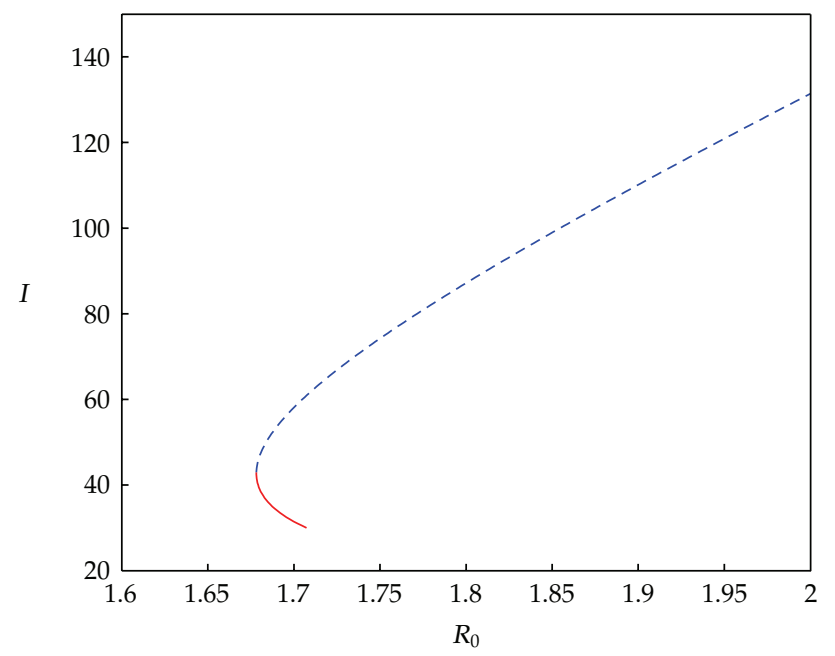

Figure 1: The figure of infective sizes at equilibria versus $R_{0}$ when $I_{0}=30, A=80, \beta_{1}=0.01, \beta_{2}=0.01$, $\gamma=0.01, d=0.9, \epsilon=0.01, r=1, p_{0}=1.6782, p_{1}=1.541, p_{2}=1.7074$, where (i) of Theorem 2.2 holds. The bifurcation from the disease-free equilibrium at $R_{0}=1$ is forward, and there is a backward bifurcation from an endemic equilibrium at $R_{0}=1$, which leads to the existence of multiple endemic equilibria.

Theorem 2.1. $E_{0}(A / d, 0,0)$ is always the disease-free equilibrium of $(1.5) . E_{*}\left(N_{*}, I_{*}, R_{*}\right)$ is an endemic equilibrium of system (1.4) if and only if $1<R_{0} \leqslant p_{2}$. Furthermore, $E_{*}$ is the unique equilibrium of system (1.4) if $1<R_{0} \leqslant p_{2}$, and one of the following conditions is satisfied:

(i) $R_{0}<p_{0}$,

(ii) $p_{0} \leqslant R_{0}<p_{1}$.

By calculation, we have $p_{2}-p_{1}=\left[d-\beta_{2}(A-k) / d\right] r-\left(\beta_{1}+\beta_{2}((\gamma+r) / d)\right) I_{0}(d+\epsilon+\gamma)$. Note that $\left[d-\beta_{2}(A-k) / d\right] r>\left(\beta_{1}+\beta_{2}(\gamma / d)\right)(d+\epsilon+\gamma) I_{0}$ is equivalent to that $p_{1}<p_{2}$.

Theorem 2.2. Endemic equilibria $E_{1}$ and $E_{2}$ do not exist if $R_{0}<p_{0}$. Further, if $R_{0} \geqslant p_{0}$, we have the following:

(i) if $\left[d-\beta_{2}(A-k) / d\right] r>\left(\beta_{1}+\beta_{2}(\gamma / d)\right)(d+\epsilon+\gamma) I_{0}$, then both $E_{1}$ and $E_{2}$ exist when $p_{1}<R_{0}<p_{2}$,

(ii) if $\left[d-\beta_{2}(A-k) / d\right] r>\left(\beta_{1}+\beta_{2}(\gamma / d)\right)(d+\epsilon+\gamma) I_{0}$, then $E_{1}$ does not exist but $E_{2}$ exists if $R_{0} \geqslant p_{2}$,

(iii) letting $\left[d-\beta_{2}(A-k) / d\right] r \leqslant\left(\beta_{1}+\beta_{2}(\gamma / d)\right)(d+\epsilon+\gamma) I_{0}$, then $E_{1}$ does not exist. Further, $E_{2}$ exists when $R_{0}>p_{2}$, and $E_{2}$ does not exist when $R_{0} \leqslant p_{2}$.

We consider $p_{0}>1$. If $\left[d-\beta_{2}(A-k) / d\right] r>\left(\beta_{1}+\beta_{2}(\gamma / d)\right)(d+\epsilon+\gamma) I_{0}$, a typical bifurcation diagram is illustrated in Figure 1, where the bifurcation from the disease-free equilibrium at $R_{0}=1$ is forward and there is a backward bifurcation from an endemic equilibrium at $R_{0}=$ 1.71 , which gives rise to the existence of multiple endemic equilibria. Further, if $\left[d-\beta_{2}(A-\right.$ $k) / d] r \leqslant\left(\beta_{1}+\beta_{2}(\gamma / d)\right)(d+\epsilon+\gamma) I_{0}$, a typical bifurcation diagram is illustrated in Figure 2, where the bifurcation at $R_{0}=1$ is forward, and (1.4) has one unique endemic equilibrium for all $R_{0}>1$. 


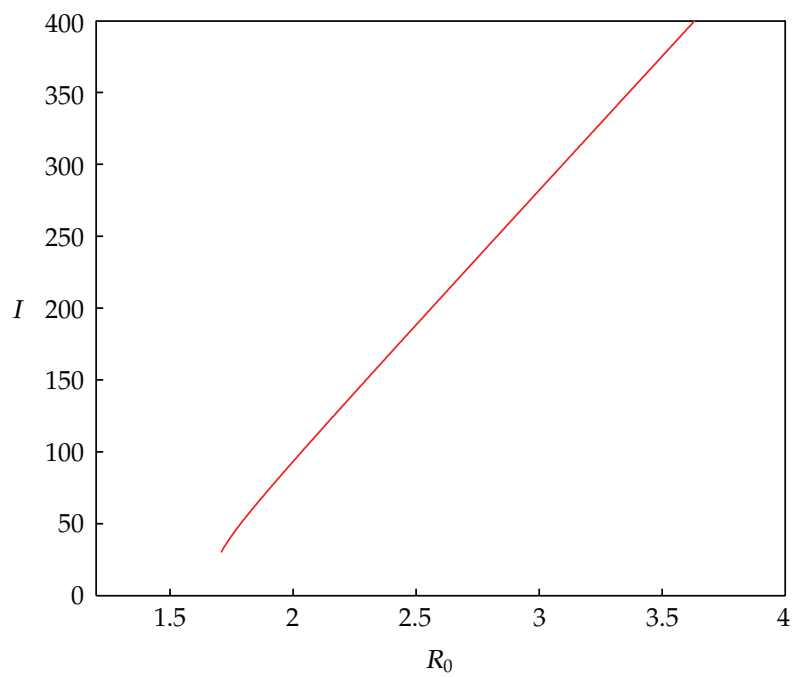

Figure 2: The diagram of $I_{*}, I_{2}$ versus $R_{0}$ when $I_{0}=30, A=100, \beta_{1}=0.01, \beta_{2}=0.01, \gamma=0.01, d=0.9$, $\epsilon=0.01, r=1, p_{0}=1.6889, p_{1}=1.7882, p_{2}=1.7074$, where (iii) of Theorem 2.2 holds. The bifurcation at $R_{0}=1$ is forward, and (1.4) has a unique endemic equilibrium for $R_{0}>1$.

Note that a backward bifurcation with endemic equilibria when $R_{0}<1$ is very interesting in applications. We present the following corollary to give conditions for such a backward bifurcation to occur.

Corollary 2.3. Equation (1.4) has a backward bifurcation with endemic equilibria when $R_{0}<1$ if $\left[d-\beta_{2}(A-k) / d\right] r>\left(\beta_{1}+\beta_{2}(\gamma / d)\right)(d+\epsilon+\gamma) I_{0}$ and $p_{0}<1$.

Example 2.4. Fix $I_{0}=10, A=60, \beta_{1}=0.01, \beta_{2}=0.005, \gamma=0.1, d=1, \epsilon=0.1$, and $r=3$. Then $p_{1} \approx 0.6779, p_{0} \approx 0.8878, p_{2} \approx 1.225$ and $\left[d-\beta_{2}(A-k) / d\right] r-\left(\beta_{1}+\beta_{2} \gamma / d\right) I_{0}(d+\epsilon+\gamma)=2.424$. Thus, (1.4) has a backward bifurcation with endemic equilibria when $R_{0}<1$ in this case (see Figure 3).

As $I_{0}$ (the capacity of treatment resources) increases, by the definition we see that $p_{0}$ increases. When $I_{0}$ is so large that $p_{0}>1$, it follows from Theorem 2.2 that there is no backward bifurcation with endemic equilibria when $R_{0}<1$. If we increase $I_{0}$ to $R_{0}<p_{0},(1.4)$ does not have a backward bifurcation because endemic equilibria $E_{1}$ and $E_{2}$ do not exist. This means that an insufficient capacity for treatment is a source of the backward bifurcation.

\section{The Stability of Equilibria}

We first determine the stability of the disease-free equilibrium $E_{0}(A / d, 0,0)$. The Jacobian matrix of $(1.4)$ at $E_{0}(A / d, 0,0)$ is

$$
\left(\begin{array}{ccc}
-d & -\epsilon & 0 \\
0 & \beta_{1} \frac{A}{d}-(d+\epsilon+\gamma+r) & \beta_{2} \frac{A}{d} \\
0 & \gamma+r & -d
\end{array}\right) .
$$




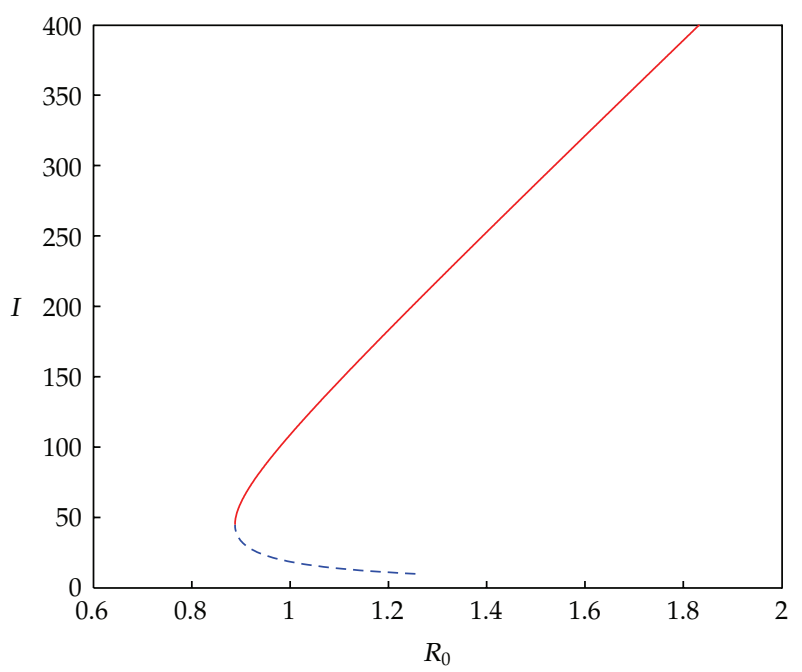

Figure 3: The figure of $I_{*}, I_{1}$ and $I_{2}$ versus $R_{0}$ that shows a backward bifurcation with endemic equilibrium when $R_{0}<1$, where Corollary 2.3 holds.

Its characteristic equation is

$$
(\lambda+d)\left[\lambda^{2}+\left(d-\beta_{1} \frac{A}{d}+(d+\epsilon+\gamma+r)\right) \lambda+d(d+\epsilon+\gamma+r) \beta_{1} \frac{A}{d}-\beta_{2} \frac{A}{d}(\gamma+r)\right]=0
$$

We obtain

$$
\begin{gathered}
\lambda_{1}=-d<0, \\
d\left[(d+\epsilon+\gamma+r)-\beta_{1} \frac{A}{d}\right]-\beta_{2} \frac{A}{d}(\gamma+r)=d(d+\epsilon+\gamma+r)\left(1-R_{0}\right) .
\end{gathered}
$$

Therefore, we get the following theorem.

Theorem 3.1. The disease-free equilibrium $E_{0}(A / d, 0,0)$ is locally asymptotically stable if $R_{0}<1$ and unstable if $R_{0}>1$.

Next, the stability of endemic equilibrium $E_{*}\left(N_{*}, I_{*}, R_{*}\right)$ is analyzed. The Jacobian matrix of $(1.4)$ at $E_{*}\left(N_{*}, I_{*}, R_{*}\right)$ is

$$
J_{*}=\left(\begin{array}{ccc}
-d & -\epsilon & 0 \\
b_{1} & \beta_{1} b_{2}-a_{1}-(d+\epsilon+\gamma+r) & \beta_{2} b_{2}-b_{1} \\
0 & \gamma+r & -d
\end{array}\right)
$$

where $c_{0}=\beta_{1} I_{*}+\beta_{2} R_{*}, b_{0}=N_{*}-I_{*}-R_{*}$. 
Making use of (2.2), the characteristic equation of $J_{*}$ is simplified into

$$
\begin{aligned}
& (\lambda+d)\left[\lambda^{2}+\left(d+c_{0}+(d+\epsilon+\gamma+r)-\beta_{1} b_{0}\right) \lambda\right. \\
& \left.\quad+d\left(c_{0}+d+\epsilon+\gamma+r-\beta_{1} b_{0}\right)+\left(c_{0}-\beta_{2} b_{0}\right)(\gamma+r)+c_{0} \epsilon\right]=0,
\end{aligned}
$$

where

$$
\begin{aligned}
& d+c_{0}+(d+\epsilon+\gamma+r)-\beta_{1} b_{0} \\
& =d+\left(\beta_{1}+\beta_{2} \frac{\gamma+r}{d}\right) I_{*}+(d+\epsilon+\gamma+r)\left(1-\frac{\beta_{1}}{\beta_{1}+\beta_{2}((\gamma+r) / d)}\right)>0, \\
& d\left(c_{0}+d+\epsilon+\gamma+r-\beta_{1} b_{0}\right)+\left(c_{0}-\beta_{2} b_{0}\right)(\gamma+r)+c_{0} \epsilon \\
& =\left(\beta_{1}+\beta_{2} \frac{\gamma+r}{d}\right) I_{*}(d+\epsilon+\gamma+r)>0 .
\end{aligned}
$$

Therefore, the real part of the all eigenvalues of $J_{*}$ is negative when $1<R_{0} \leqslant p_{2}$.

Theorem 3.2. If $1<R_{0} \leqslant p_{2}$, then the endemic equilibrium $E_{*}$ of (1.4) is locally asymptotically stable.

Afterwards, we study the stability of endemic equilibrium $E_{1}\left(N_{1}, I_{1}, R_{1}\right)$. The characteristic equation of Jacobian matrix of (1.4) at $E_{1}\left(N_{1}, I_{1}, R_{1}\right)$ is

$$
(\lambda+d)\left[\lambda^{2}+\left(d+c_{1}+(d+\epsilon+\gamma)-\beta_{1} b_{1}\right) \lambda+d\left(c_{1}+d+\epsilon+\gamma-\beta_{1} b_{1}\right)+\gamma\left(c_{1}-\beta_{2} b_{1}\right)+c_{1} \epsilon\right]=0
$$

where $c_{1}=\beta_{1} I_{1}+\beta_{2} R_{1}, b_{1}=N_{1}-I_{1}-R_{1}$. After some calculations, we obtain

$$
d\left(c_{1}+d+\epsilon+\gamma-\beta_{1} b_{1}\right)+\gamma\left(c_{1}-\beta_{2} b_{1}\right)+c_{1} \epsilon=2 a_{0} I_{1}+a_{1}=-\sqrt{\Delta}<0
$$

Therefore, (3.7) has positive real part eigenvalues. Thus $E_{1}\left(N_{1}, I_{1}, R_{1}\right)$ is unstable.

Theorem 3.3. If the endemic equilibrium $E_{1}\left(N_{1}, I_{1}, R_{1}\right)$ of system (1.4) exists, then it is unstable. equation is

Finally, we analyze the stability of endemic equilibrium $E_{2}\left(N_{2}, I_{2}, R_{2}\right)$. Its characteristic

$$
(\lambda+d)\left[\lambda^{2}+\left(d+c_{2}+(d+\epsilon+\gamma)-\beta_{1} b_{2}\right) \lambda+d\left(c_{2}+d+\epsilon+\gamma-\beta_{1} b_{2}\right)+\gamma\left(c_{2}-\beta_{2} b_{2}\right)+c_{2} \epsilon\right]=0
$$


where $c_{2}=\beta_{1} I_{2}+\beta_{2} R_{2}, b_{2}=N_{2}-I_{2}-R_{2}$. By some calculations, we obtain

$$
\begin{aligned}
d\left(c_{2}+\right. & \left.d+\epsilon+\gamma-\beta_{1} b_{2}\right)+\gamma\left(c_{2}-\beta_{2} b_{2}\right)+c_{2} \epsilon \\
& =2 a_{0} I_{2}+a_{1}=\sqrt{\Delta}>0 \\
d+c_{2} & +(d+\epsilon+\gamma)-\beta_{1} b_{2} \\
= & d+\left(\beta_{1}+\beta_{2} \frac{\gamma}{d}\right) I_{2}+(d+\epsilon+\gamma)+\frac{\beta_{2} k}{d}+\frac{\beta_{1}(d+\epsilon+\gamma) I_{2}}{d}+\frac{\beta_{1}(k-A)}{d} .
\end{aligned}
$$

It follows that $d+c_{2}+(d+\epsilon+\gamma)-\beta_{1} b_{2}>0$ is equivalent to

$$
\sqrt{\Delta}>a_{1}+\frac{-2 a_{0}\left[d+\beta_{2} k / d+(d+\gamma+\epsilon)-\beta_{1}((A-k) / d)\right]}{\beta_{1}+\beta_{2}(\gamma / d)+\beta_{1}((d+\gamma+\epsilon) / d)} .
$$

If

$$
a_{1}\left[\beta_{1}+\beta_{2} \frac{\gamma}{d}+\beta_{1} \frac{d+\gamma+\epsilon}{d}\right]-2 a_{0}\left[d+\frac{\beta_{2} k}{d}+(d+\gamma+\epsilon)-\beta_{1} \frac{A-k}{d}\right]<0,
$$

then $d+c_{2}+(d+\epsilon+\gamma)-\beta_{1} b_{2}>0$. Thus $E_{2}\left(N_{2}, I_{2}, R_{2}\right)$ is locally asymptotically stable.

By complicated calculation, if $a_{1}\left[\beta_{1}+\beta_{2}(\gamma / d)+\beta_{1}((d+\gamma+\epsilon) / d)\right]-2 a_{0}\left[d+\beta_{2} k / d+\right.$ $\left.(d+\gamma+\epsilon)-\beta_{1}((A-k) / d)\right]>0$, then (3.11) is equivalent to

$$
\begin{aligned}
a_{2}\left[\beta_{1}+\beta_{2} \frac{\gamma}{d}+\beta_{1} \frac{d+\gamma+\epsilon}{d}\right]^{2}< & a_{1}\left[\beta_{1}+\beta_{2} \frac{\gamma}{d}+\beta_{1} \frac{d+\gamma+\epsilon}{d}\right]\left[d+\frac{\beta_{2} k}{d}+(d+\gamma+\epsilon)-\beta_{1} \frac{A-k}{d}\right] \\
& -a_{0}\left[d+\frac{\beta_{2} k}{d}+(d+\gamma+\epsilon)-\beta_{1} \frac{A-k}{d}\right]^{2} .
\end{aligned}
$$

Theorem 3.4. Suppose the endemic equilibrium $E_{2}\left(N_{2}, I_{2}, R_{2}\right)$ of system (1.4) exists; if either

$$
a_{1}\left[\beta_{1}+\beta_{2} \frac{\gamma}{d}+\beta_{1} \frac{d+\gamma+\epsilon}{d}\right]-2 a_{0}\left[d+\frac{\beta_{2} k}{d}+(d+\gamma+\epsilon)-\beta_{1} \frac{A-k}{d}\right]<0,
$$

or

$$
\begin{gathered}
a_{1}\left[\beta_{1}+\beta_{2} \frac{\gamma}{d}+\beta_{1} \frac{d+\gamma+\epsilon}{d}\right]-2 a_{0}\left[d+\frac{\beta_{2} k}{d}+(d+\gamma+\epsilon)-\beta_{1} \frac{A-k}{d}\right]>0, \\
a_{2}\left[\beta_{1}+\beta_{2} \frac{\gamma}{d}+\beta_{1} \frac{d+\gamma+\epsilon}{d}\right]^{2}<a_{1}\left[\beta_{1}+\beta_{2} \frac{\gamma}{d}+\beta_{1} \frac{d+\gamma+\epsilon}{d}\right]\left[d+\frac{\beta_{2} k}{d}+(d+\gamma+\epsilon)-\beta_{1} \frac{A-k}{d}\right] \\
-a_{0}\left[d+\frac{\beta_{2} k}{d}+(d+\gamma+\epsilon)-\beta_{1} \frac{A-k}{d}\right]^{2},
\end{gathered}
$$

then it is locally asymptotically stable. 


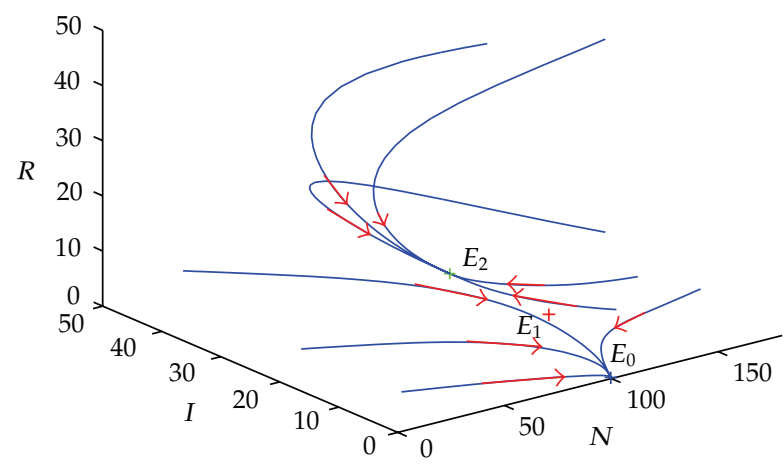

Figure 4: The phase diagram of system (1.4) when $I_{0}=5, A=80, \beta_{1}=0.015, \beta_{2}=0.001, \gamma=0.01, d=0.8$, $\epsilon=0.01, r=1, p_{0}=0.8607, p_{1}=0.6589, p_{2}=1.1016, R_{0}=0.8935$.

Theorem 3.5. The disease-free equilibrium $E_{0}$ of system (1.4) is globally asymptotically stable, if one of the following conditions is satisfied:

(i) $R_{0}<1$ and $p_{0}>1$,

(ii) $R_{0}<1, p_{0}<1$ and $p_{1} \geqslant 1$.

Proof. $R_{0}<1$ implies that $E_{*}$ does not exist. Suppose $p_{0} \geqslant 1$. It follows from the discussions for Theorem 2.2 that $E_{1}$ or $E_{2}$ exists only if $R_{0}>p_{0}$, which is impossible since we have $R_{0}<1$. Let us now suppose $p_{0}<1$ and $p_{1} \geqslant 1$. If $\left[d-\beta_{2}(A-k) / d\right] r>\left(\beta_{1}+\beta_{2}(\gamma / d) I_{0}(d+\epsilon+\gamma)\right.$, since $p_{1}<$ $p_{2}$, it follows from the discussions for (i), (ii) of Theorem 2.2 that $E_{1}$ or $E_{2}$ exists only if $R_{0}>p_{1}$, which is impossible since we have $R_{0}<1$. If $\left[d-\beta_{2}(A-k) / d\right] r>\left(\beta_{1}+\beta_{2} \gamma / d\right) I_{0}(d+\epsilon+\gamma)$, since $1<p_{2}$, it follows from (iii) of Theorem 2.2 that $E_{1}$ and $E_{2}$ do not exist. In summary, endemic equilibria do not exist under the assumptions.

\section{The Simulation of Model}

In this section, we give the numerical simulations of system (1.4) for the conclusions gained previously.

Example 4.1. For system (1.4), if $R_{0}<1$ and $R_{0}>p_{0}$ and $p_{1}<R_{0}<p_{2}$, then the equilibrium $E_{*}$ does not exist, and there are three equilibria $E_{0}, E_{1}$, and $E_{2}$. Its phase diagram is illustrated in Figure 4. Numerical calculations show that $E_{0}$ and $E_{2}$ are stable, but $E_{1}$ is unstable.

Example 4.2. For system (1.4), if $R_{0}>1$ and $R_{0}<p_{0}$, there is the unique equilibrium $E_{*}$ which is stable. Its phase diagram is illustrated in Figure 5. Numerical calculations show that the unique equilibrium $E_{*}$ is globally stable.

Example 4.3. For system (1.4), if $R_{0}>1$ and $R_{0}>p_{0}$ and $p_{1}<R_{0}<p_{2}$, the equilibria $E_{2}$ and $E_{*}$ are stable, and $E_{0}$ and $E_{1}$ are unstable; its phase diagram is illustrated in Figure 6. Numerical calculations show that the equilibria $E_{2}$ and $E_{*}$ are stable, and $E_{0}$ and $E_{1}$ unstable. Thus, we have bistable endemic equilibria. 


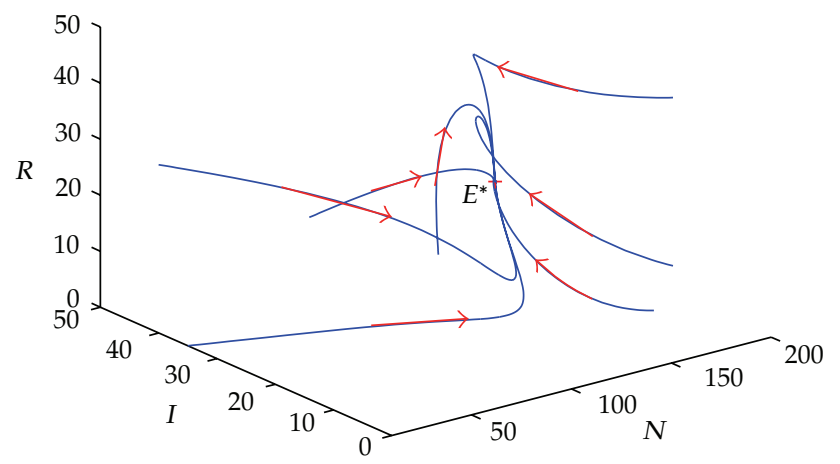

Figure 5: The phase diagram of system (1.4) when $I_{0}=40, A=100, \beta_{1}=0.02, \beta_{2}=0.01, \gamma=0.01, d=0.9$, $\epsilon=0.01, r=1.5, p_{0}=2.6340, p_{1}=2.6606, p_{2}=2.6346, R_{0}=1.6886$.

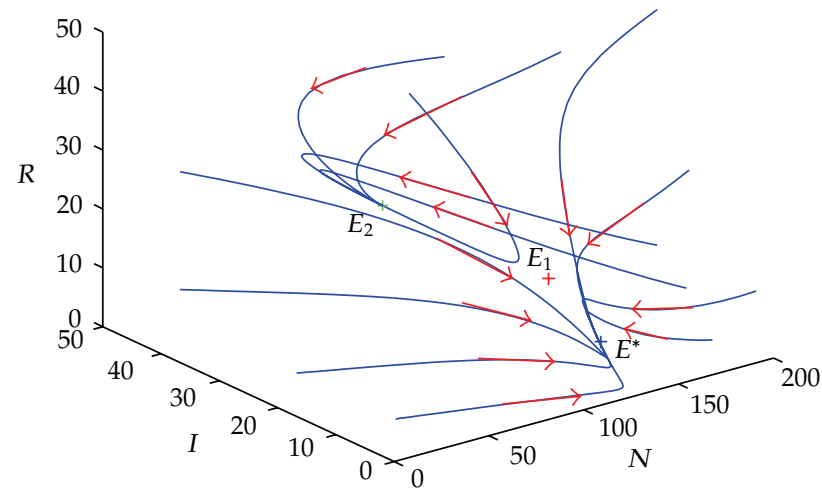

Figure 6: The phase diagram of system (1.4) when $I_{0}=10, A=100, \beta_{1}=0.015, \beta_{2}=0.001, \gamma=0.01, d=0.8$, $\epsilon=0.01, r=1, p_{0}=1.0462, p_{1}=0.8156, p_{2}=1.2033, R_{0}=1.1169$.

\section{Discussion}

In this paper, we have proposed an epidemic model with infectious force in infected and immune period and treatment rate of infectious individuals to understand the effect of the capacity for treatment of infective on the disease transmission, which can occur when patients have to be hospitalized but there are limited beds or medical establishments in hospitals, or there is not enough medicine for treatment. We have shown in Theorem 2.2 and Corollary 2.3 that backward bifurcations occur because of the insufficient capacity for treatment. We have also shown that system (1.4) has bistable endemic equilibria because of the limited resources. This means that the basic reproduction number $R_{0}<1$ and small treatment rate are not enough to eradicate the disease, but the basic reproduction number $R_{0}<1$ and large treatment rate may eradicate the disease. The disease cannot be eradicated for any treatment rate if the basic reproduction number $R_{0}>1$. Therefore, the level of initial infectious invasion must be lowered to a threshold so that the disease dies out or approaches a lower endemic steady state for a range of parameters.

In Sections 2 and 3, when $I>I_{0}$, with respect to the existence and the local stability of the endemic equilibrium we only proved for the model (1.6) under the restriction $a_{2}>0$. But the case of $a_{2}<0$ is an unsolved question. 


\section{Acknowledgment}

This work is supported by the National Science Foundation of China (10471040), Science Foundation of China (2009011005-1) as well as Science and Technology Research Developmental item of Shan xi Province Education Department (20061025).

\section{References}

[1] J. Zhang and Z. E. Ma, "Global analysis of the SEI epidemic model with constant inflows of different compartments," Journal of Xi'an Jiaotong University, vol. 37, no. 6, pp. 653-656, 2003.

[2] S. L. Yuan, L. T. Han, and Z. E. Ma, "A kind of epidemic model having infectious force in both latent period and infected period," Journal of Biomathematics, vol. 16, no. 4, pp. 392-398, 2001.

[3] G. H. Li and Z. Jin, "Global stability of an SEI epidemic model," Chaos, Solitons and Fractals, vol. 21, no. 4, pp. 925-931, 2004.

[4] G. H. Li and Z. Jin, "Global stability of an SEI epidemic model with general contact rate," Chaos, Solitons and Fractals, vol. 23, no. 3, pp. 997-1004, 2005.

[5] G. H. Li and Z. Jin, "Global stability of a SEIR epidemic model with infectious force in latent, infected and immune period," Chaos, Solitons and Fractals, vol. 25, no. 5, pp. 1177-1184, 2005.

[6] J. Arino, C. C. McCluskey, and P. van den Driessche, "Global results for an epidemic model with vaccination that exhibits backward bifurcation," SIAM Journal on Applied Mathematics, vol. 64, no. 1, pp. 260-276, 2003.

[7] J. Dushoff, W. Huang, and C. Castillo-Chavez, "Backwards bifurcations and catastrophe in simple models of fatal diseases," Journal of Mathematical Biology, vol. 36, no. 3, pp. 227-248, 1998.

[8] P. van den Driessche and J. Watmough, "A simple SIS epidemic model with a backward bifurcation," Journal of Mathematical Biology, vol. 40, no. 6, pp. 525-540, 2000.

[9] K. P. Hadeler and P. van den Driessche, "Backward bifurcation in epidemic control," Mathematical Biosciences, vol. 146, no. 1, pp. 15-35, 1997.

[10] Y. A. Kuznetsov and C. Piccardi, "Bifurcation analysis of periodic SEIR and SIR epidemic models," Journal of Mathematical Biology, vol. 32, no. 2, pp. 109-121, 1994.

[11] S. Ruan and W. Wang, "Dynamical behavior of an epidemic model with a nonlinear incidence rate," Journal of Differential Equations, vol. 188, no. 1, pp. 135-163, 2003.

[12] X. Zhang and X. Liu, "Backward bifurcation of an epidemic model with saturated treatment function," Journal of Mathematical Analysis and Applications, vol. 348, no. 1, pp. 433-443, 2008.

[13] Z. Hu, S. Liu, and H. Wang, "Backward bifurcation of an epidemic model with standard incidence rate and treatment rate," Nonlinear Analysis. Real World Applications, vol. 9, no. 5, pp. 2302-2312, 2008.

[14] X.-Z. Li, W.-S. Li, and M. Ghosh, "Stability and bifurcation of an SIR epidemic model with nonlinear incidence and treatment," Applied Mathematics and Computation, vol. 210, no. 1, pp. 141-150, 2009.

[15] X. Zhang and X. Liu, "Backward bifurcation and global dynamics of an SIS epidemic model with general incidence rate and treatment," Nonlinear Analysis. Real World Applications, vol. 10, no. 2, pp. 565 $575,2009$.

[16] W. Wang and S. Ruan, "Bifurcation in an epidemic model with constant removal rate of the infectives," Journal of Mathematical Analysis and Applications, vol. 291, no. 2, pp. 775-793, 2004.

[17] W. Wang, "Backward bifurcation of an epidemic model with treatment," Mathematical Biosciences, vol. 201, no. 1-2, pp. 58-71, 2006. 


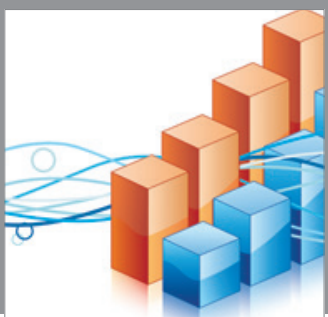

Advances in

Operations Research

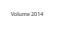

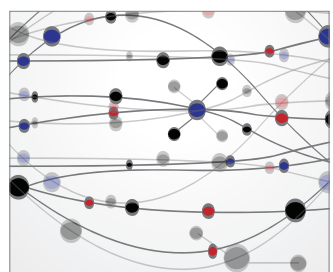

\section{The Scientific} World Journal
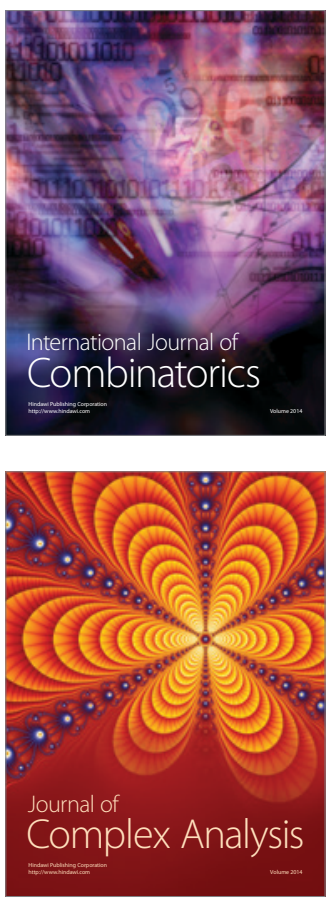

International Journal of

Mathematics and

Mathematical

Sciences
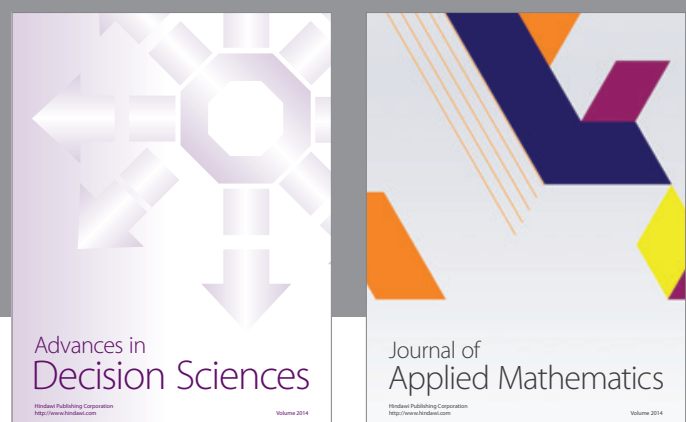

Journal of

Applied Mathematics
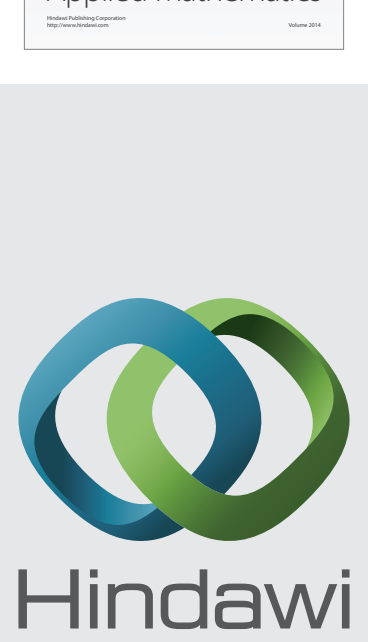

Submit your manuscripts at http://www.hindawi.com
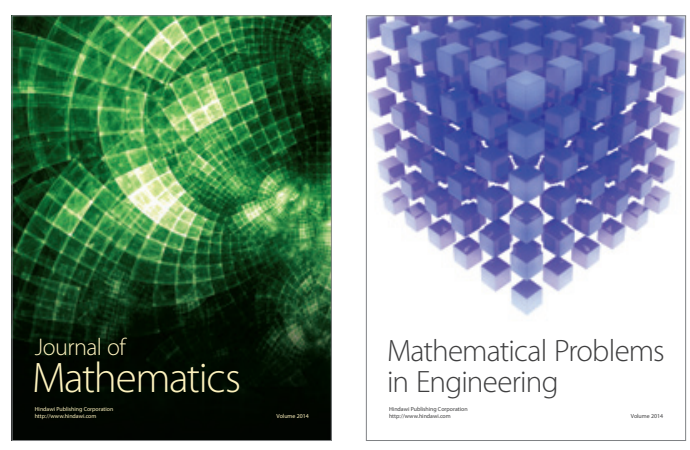

Mathematical Problems in Engineering
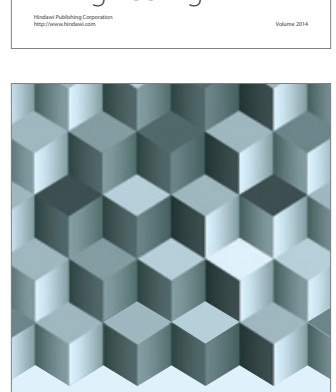

Journal of

Function Spaces
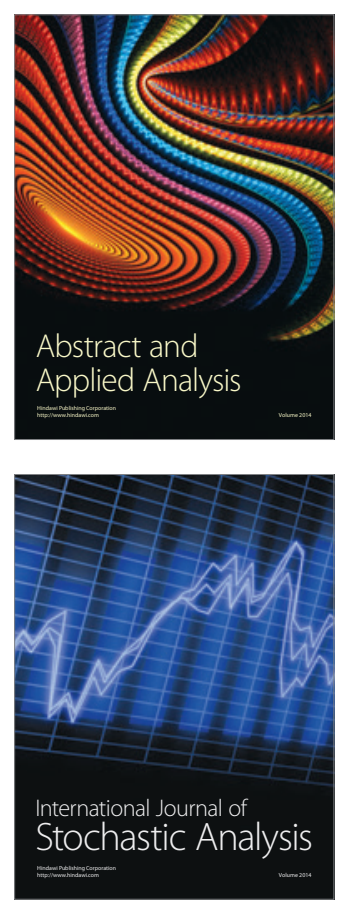

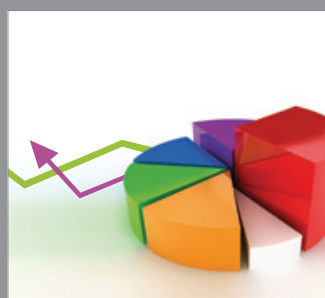

ournal of

Probability and Statistics

Promensencen
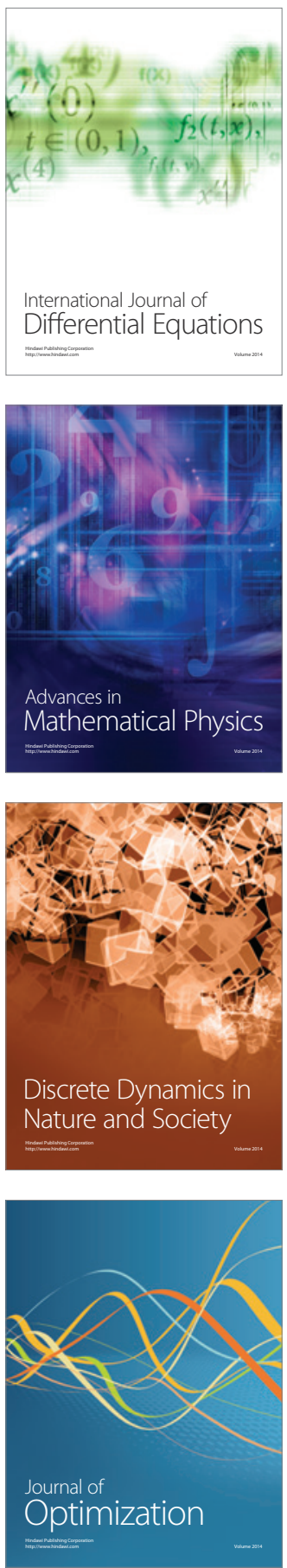